\title{
REVISIONES
}

\section{La investigación como forma de desarrollo profesional docente: Retos y perspectivas}

\author{
Research as a means of teachers' professional development: \\ Challenges and perspectives \\ Pesquisas como uma forma de professor desenvolvimento \\ profissional: Desafios e perspectivas
}

\author{
Muñoz Martínez, Maryluz ${ }^{a}$ Garay Garay, Fredy ${ }^{b}$ \\ aUniversidad Pedagógica Nacional, Bogotá, Colombia. Teléfono: 300-5657773. \\ Correo electrónico: mary-mz192011@hotmail.com

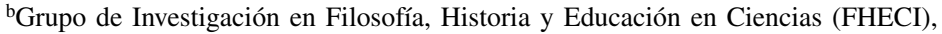 \\ Universidad Católica de Colombia - Universidad Pedagógica Nacional, Bogotá, Colombia. \\ Teléfono: 300-7376881. \\ Correo electrónico: licfredygaray@ gmail.com
}

\section{RESUMEN}

Las reflexiones aquí presentadas visan la importancia de la investigación educativa como proceso permanente de desarrollo profesional, que permite el empoderamiento de la profesión docente, los conocimientos que caracterizan al docente investigador, las limitaciones que emergen durante dicho proceso investigativo y las estrategias desarrolladas en diferentes países para lograr resultados que impacten en el aprendizaje de los profesores y, por tanto, les permita transformar sus prácticas profesionales para que ello redunde positivamente en la enseñanza a los educandos.
\end{abstract}

Palabras clave: investigación educativa, profesional docente, enseñanza, aprendizaje.

\section{ABSTRACT}

The reflections presented here remark the importance of educational research as an ongoing process of professional development. As understood, this process enables the empowerment of the teaching profession, the knowledge that characterizes the teacher and researcher, the limitations that emerge during the research process, and the strategies developed in different countries to achieve results that have an impact on the learning of teachers; thus permitting them to transform their professional practice resulting on positive effects on those been taught.

Key words: educational research, teacher professional, teaching, learning.

\section{RESUMO}

As reflexões aqui apresentadas visam à importância da pesquisa educativa como permanente processo de desenvolvimento profissional docente. Nesse sentido, tal processo permite o empoderamento da profissão docente, dos conhecimentos que caracterizam o docente pesquisador, das limitações que surgem durante o processo de pesquisa e das estratégias desenvolvidas em diferentes países para alcançar resultados que impactam sobre a aprendizagem dos professores permitindo-lhes transformar suas práticas profissionais, o que resulta positivamente na aprendizagem dos estudantes.

Palavras chave: pesquisa educacional, profissional docente, ensino, aprendizagem. 


\section{LA INVESTIGACIÓN EDUCATIVA Y EL PAPEL DEL PROFESOR}

La educación está permeada por contextos como el social, económico y político inmersos en la cultura, esto obliga a que los procesos de enseñanza y aprendizaje respondan a las nuevas dinámicas de la sociedad actual, a sus necesidades y claramente a las expectativas generadas por procesos como la globalización.

Para dar cuenta de las diferentes problemáticas que se presentan en los sistemas educativos y en el proceso mismo de la educación, se acude a la investigación educativa, entendida esta como proceso en el cual el investigador se cuestiona sobre un problema o situación de tipo educativo, lo define, analiza, formula acciones para mejorar y estas a su vez pueden ser aplicadas en las aulas de clase con el fin de provocar cambios relevantes (Martínez González, 2007; Imbernón et al., 2007). Como lo afirma Martínez González, la investigación es una acción cada vez más necesaria para identificar y diagnosticar necesidades educativas, sociales, institucionales y personales, y para promover cambios eficaces en las prácticas educativas, de enseñanza, en la organización de los centros e instituciones educativas, en los procesos de convivencia y resolución de conflictos y en las relaciones que mantienen los diversos agentes de la comunidad educativa (2007: 7).

La investigación en educación es un proceso cada vez más indispensable para renovar y transformar los ambientes escolares, de enseñanza y aprendizaje logrando calidad en la educación, es decir, que responda a las necesidades de los estudiantes según sus contextos. Entonces, la investigación se convierte en un factor importante y necesario en los entornos escolares, permitiendo transformaciones favorables, "es decir, la investigación debe proporcionar nuevas y mejores formas de comprensión sobre la dinámica de los procesos de enseñanza y aprendizaje" (Cit. en Kennedy, 1997: 10), por lo que la mejora de los procesos y resultados educativos debe ser el principal propósito de la investigación educativa. En este contexto, "la investigación educativa tiene un doble objetivo: la producción de conocimiento y la mejora de la práctica docente" (Cit. en Vanderlinde \& Braak, 2010: 299).

La pretensión para el cumplimiento de estos dos objetivos conduce al reclamo en diversos estudios (Kennedy, 1997; Hiebert, Gallimore \& Stigler, 2002; Lüdke, 2005; Miretzky, 2007; Schoonmaker, 2007; Cochran-Smith \& Lytle, 2009) de una mayor inclusión de los profesores en la investigación educativa, en la que se concibe al profesional de la enseñanza como líder innovador, que tiene a su alcance las herramientas de tipo conceptual y práctico para pensar y actuar frente a aquello que la educación demanda.

Esta investigación se asume como una forma posible para el desarrollo profesional, "en la medida en que puede relacionarse con la práctica docente y con los profesores que se encuentran inmersos en tales procesos de investigación" (Cit. en Maciel de Oliveira, 2003: 1), por lo que una práctica profesional docente solicita profesores con un fuerte compromiso educativo, conscientes de la responsabilidad social que conlleva la enseñanza, competentes y autónomos en la toma de decisiones, dispuestos al cambio, con dominio de conocimientos en lo disciplinar, didáctico y pedagógico, con cualidades éticas y valores que lo distingan de otros profesionales, líderes críticos para transformar su quehacer profesional y la escuela a la vez, empeñados en aprender mediante la formación continua, con capacidad para el trabajo en equipo y, con habilidades y destrezas para la investigación. 
En este sentido, Cochran-Smith \& Lytle "muestran el rol del profesor como tomador de decisiones, consultor, desarrollador del currículo, analista, activista, líder escolar, y también como conocedor de los cambios en los contextos escolares" (2009: 4). Esto transforma el rol del profesor pasando de ser un mero consumista de los productos de las investigaciones, y lo convierte en un ente activo de dichos procesos y resultados.

Son bastantes las cualidades, conocimientos, actitudes y aptitudes que se esperan de los profesores, que permitan posicionarlos en la sociedad como gestores de verdaderos cambios educativos y sociales. Desde el siglo pasado se ha estudiado los beneficios de la investigación para que el profesor adquiera y desarrolle saberes propios de su profesión y los aplique en su contexto.

Cochran-Smith \& Lytle plantean que "la idea de emplear los resultados de las investigaciones en las aulas de clase está ligada a programas de desarrollo profesional y estrategias de profesionalización docente, al mejoramiento escolar y curricular procurando cambios estructurados y organizados" (2009: 4).

El énfasis es transformar la teoría y la práctica educativa hacia un fin emancipador, transformando la práctica profesional y la investigación educativa en formas de empoderamiento, en la que los profesores generan conocimiento en y sobre la enseñanza, el aprendizaje y la escolaridad, cuando convierten sus salones de clase, en espacios y proyectos de investigación, aprovechando el trabajo colaborativo entre comunidades de investigación.

Reconocer que la investigación educativa es un proceso que mejora la enseñanza y genera conocimiento, debe estimular al profesor para que sea una práctica recurrente en su quehacer profesional. Investigaciones evidencian que "las actividades desarrolladas por los alumnos poseen características rutinarias y repetitivas, alejadas del entorno sociocultural y los saberes previos de los alumnos, lo cual no favorece el aprendizaje" (2004: 8), por lo que los profesores deben involucrarse como agentes activos en las investigaciones para superar las dificultades que se presentan en las aulas de clase.

Esto se puede lograr mediante el análisis, la reflexión y el actuar frente a las múltiples problemáticas que se presenta en los contextos escolares, el docente debe enfrentar los retos que exigen los niños, niñas y jóvenes con los que se trabaja a diario. Como lo afirman Cochran-Smith \& Lytle (2009: 3), "los profesores podrían ser poderosos agentes en la escena educativa, capaces de hacer una diferencia en virtud de las decisiones que pueden tomar basadas en el día a día".

Analizar y reflexionar propicia el repensar sobre el para qué enseñar cierta disciplina y también acerca de los procedimientos y fundamentos usados que permiten que los alumnos aprendan. Por su parte, actuar es poner en práctica conocimientos construidos y adquiridos en la práctica, según Schoonmaker, "la investigación deberá elaborar los problemas de la práctica y ayudar a los profesores en la toma de decisiones", sin embargo, se debe evidenciar cuales son los tipos de conocimiento que caracterizan un profesional de la educación, para de esta manera, poder facilitar tales procesos de investigación (Schoonmaker, 2007: 266).

\section{DEL CONOCIMIENTO DEL PROFESOR}

Identificar los saberes del profesor, se hace relevante para establecer las características básicas que pueden diferenciar al profesor investigador del experto y del novato. Además, 
para transformar y optimizar los procesos de enseñanza en el aula, "la profesión docente necesita una base de conocimientos que crece y mejora" (Hiebert et al., 2002: 3).

Kennedy (2002) plantea tres conocimientos básicos en la enseñanza: el artesanal, el prescriptivo y el sistemático. El primero se asocia en mayor medida al adquirido con la experiencia, el segundo relacionado con las políticas educativas e institucionales y el tercero se relaciona con lo aprendido en investigaciones, estudios profesionales y comunidades de especialistas, entre otros. La dinámica de los procesos educativos obliga a que existan interrelaciones entre estos conocimientos, los que deben ser adaptativos y actualizados.

Hiebert et al. (2002), al igual que Kennedy (2002), caracterizan tres tipos de conocimiento: profesional, base y práctico.

El conocimiento profesional se estructura por la formación en la disciplina, la historia, epistemología, normas y leyes educativas; mientras el conocimiento base reúne los componentes didáctico, pedagógico, y establece las relaciones con la disciplina básica y finalmente el conocimiento práctico se refiere al componente actitudinal, procedimental y actuacional (Garay, 2013: 18).

Al igual que en la propuesta de Kennedy (2002), no es posible dejar de lado el carácter relacional de estos tres tipos de conocimientos, ya que es este carácter el que posibilita las transformaciones de los procesos de enseñanza y aprendizaje.

Al profesor investigador lo debe distinguir su habilidad y pericia para hacer posible en su trabajo cotidiano el dominio de los conocimientos profesional, base y práctico. Mediante la formación en el pregrado, especializaciones, posgrado, cursos y diplomados, la participación en investigaciones y en eventos académicos, el profesor estructura unas bases conceptuales que le permiten construir de forma articulada su conocimiento profesional relacionándolo con su conocimiento base y práctico por medio de dicha preparación continua y reflexiva.

El conocimiento base estará ligado a las bases conceptuales del área del conocimiento específica y a su didáctica, que posibilitan de manera más eficaz trabajar en el contexto educativo un determinado saber, ya que el profesor transforma el conocimiento especializado en conocimiento escolar. El conocimiento base y práctico se vincula gracias a la reflexión y planificación del quehacer diario en las aulas de clase.

El conocimiento práctico se constituye de las acciones del educador que serán guiadas por sus actitudes y forma de proceder frente a los diferentes sucesos que puedan generarse en el contexto específico educativo, se correlaciona con los conocimientos profesional y base mediante la praxis y el aprendizaje que se obtiene por el desarrollo de la labor educativa (ver Figura 1).

En cualquiera de los casos es preciso reconocer el contexto del estudiante, de la institución, y en general de la sociedad en la que se está inmerso, convirtiendo la labor realizada en pertinente y aplicada a las necesidades sociales que emergen. Estos conocimientos esenciales del profesor demanda de ellos, en primera instancia una formación profesional que apunte al aprendizaje de los componentes disciplinar, didáctico y pedagógico enmarcados dentro de la investigación y una práctica profesional reflexiva. Según Chi, "en la práctica reflexiva, los profesores deben ser alentados a prestar más atención a la reflexión con respecto a la forma en que enseñan y cómo involucrar a los estudiantes en las actividades" (2010: 182) y cómo tales actividades son asertivas o por el contrario deben ser reformuladas. 
Figura 1. Relaciones entre los conocimientos: profesional, base y práctico

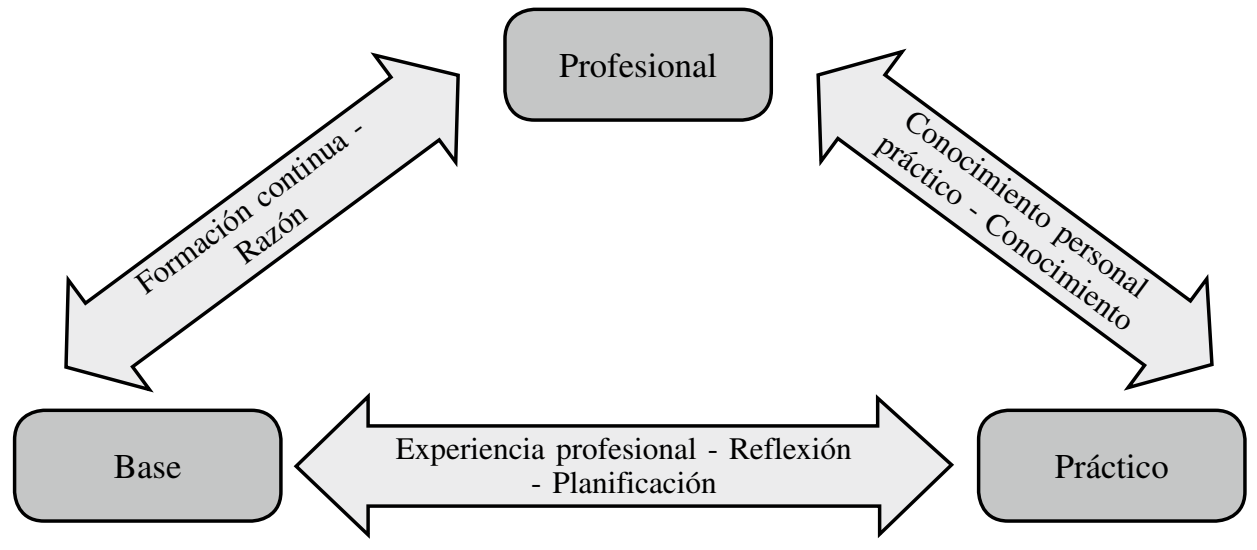

Es así, que la reflexión puede ser asumida como un "proceso de compromiso con la enseñanza que brinda la oportunidad de un análisis crítico y una evaluación continua, con el fin de mejorar las prácticas educativas" (Black \& Plowright, 2010: 246), lo que le permite al educador realizar conexiones entre sus diferentes conocimientos para que sean transformadas ciertamente sus prácticas profesionales favoreciendo los procesos generados en el interior de las aulas de clase, gracias al aprendizaje de conocimientos propios del ejercicio docente como a su aplicación en la enseñanza. De acuerdo con Chi "este proceso obliga a los profesores a adoptar una actitud autocrítica y desafiar a sus propias creencias personales sobre la enseñanza y el aprendizaje" (2010: 172).

También, la colaboración es un proceso fundamental para el éxito profesional porque garantiza la comunicación y el trabajo en equipo para posibilitar "que lo que se descubrió será transmisible, ya que se hizo en el contexto de la discusión en grupo, el debate, la verificación y la refutación o modificación" (Hiebert et al., 2002: 7). La colaboración facilita la mejora de la educación mediante el intercambio dialógico de saberes, por ejemplo, en las comunidades de aprendizaje diferentes profesionales y estudiantes con una meta en común interaccionan para cuestionar, razonar, discutir y generar conocimiento acerca de temas relevantes de la educación. La colaboración se enfoca a ir más allá de un aporte de cada miembro del grupo, en estos casos, el objetivo u objetivos propuestos permiten la cohesión de cada integrante para llegar a un punto en común gracias al trabajo mancomunado de la comunidad. Además, como lo plantean Meirink, Imants, Meijer \& Verloop (2010), la colaboración entre equipos es útil como entorno de aprendizaje y para diseñar y experimentar con nuevas prácticas de enseñanza.

\section{LIMITACIONES DE LA INVESTIGACIÓN EDUCATIVA}

Es notoria la distancia entre práctica docente e investigación educativa, ya que esta última, como lo afirman Kennedy (1997), Schoonmaker (2007), Vanderlinde \& Braak (2010) no ha sido relevante para la práctica, es limitada en su uso práctico, los estudios no han sido lo suficientemente impactantes como para proporcionar resultados convincentes, 
no proporciona resultados válidos y fiables, no se ha ocupado de las preguntas de los profesores, se usa poco o inadecuadamente en el ejercicio profesional.

Los resultados de las investigaciones no se han expresado de manera que sean comprensibles para los profesores y adicionalmente, los profesores se resisten a involucrarse en ellas (Miretzky, 2007), porque les demanda esfuerzos que no se quieren asumir, las instituciones para las que se trabaja no apoyan verdaderamente estos procesos, los maestros tienen poco tiempo para leer y aplicar la investigación incluso si son apoyados, o porque resulta poco interesante aplicarlas, ya que no se relacionan con los contextos y necesidades particulares (Kennedy, 1997; Schoonmaker, 2007; Vanderlinde \& Braak, 2010).

Otra limitante en la investigación educativa es la poca inclusión en los planes de estudio del pregrado de las suficientes disciplinas específicas sobre formación en investigación y la falta de posibilidad de participación en la investigación universitaria (Lüdke, 2005) o si se cuenta con asignaturas específicas en investigación, el enfoque que se da a estas no es el pertinente para que los estudiantes aprendan en dicho proceso y que a su vez esto constituya un eje articulador del ejercicio docente.

Las instituciones educativas que forman maestros deben reflexionar sobre la trascendencia de los programas ofrecidos, dando mayores espacios reflexivos en sus currículos a la investigación como una forma eficaz de evitar el futuro fracaso de los profesores en las escuelas. Según Lüdke, se demanda de la universidad, especialmente, "la formación de profesionales con un nuevo perfil, capaces de dar respuestas más adecuadas a los problemas que enfrenta el sistema escolar, a través del trabajo en investigación y discutiendo la estructura misma del conocimiento en sus diferentes ámbitos, impulsando a los jóvenes a cuestionarse constantemente" (2005: 346).

Se deben reunir varias condiciones para que sea exitosa la misión de las instituciones de educación superior en la formación de profesionales de la educación, por ejemplo, profesores que promuevan y creen grupos de investigación; asignaturas que procuren el aprendizaje de conocimientos relacionados con epistemología, perspectivas teóricas, metodologías y métodos de investigación; prácticas pedagógicas donde se apliquen estos conocimientos teóricos, en los que se practique un ejercicio profesional autónomo y reflexivo; vínculos formativos entre docentes de educación primaria, secundaria, pregrado y posgrado; fomento en la participación de actividades académicas nacionales e internacionales, entre otros.

\section{LA INVESTIGACIÓN EN LOS CURRÍCULOS DE LAS UNIVERSIDADES FORMADORAS DE PROFESORES EN CIENCIAS DE BOGOTÁ}

Para estar a la vanguardia con las tendencias de la educación, las instituciones educativas requieren de profesores investigadores que lideren cambios y propongan estrategias que impacten de forma asertiva las comunidades escolares y la sociedad en la cual están inmersos.

Para ello, los programas de las universidades que forman a los profesores deben ofrecer a sus educandos en los currículos, un enfoque de investigación educativa permanente que trascienda en las aulas de clase, provocando nuevas formas de pensar, centrada en un perfil de educador como innovador que genera conocimiento en sus prácticas pedagógicas, que comparte, discute y se cuestiona sobre los problemas de la educación. Las 
universidades deben combinar elementos disciplinares, didácticos y pedagógicos junto a metodologías de investigación que permitan la construcción de conocimiento teórico y práctico, recobrándose la importancia del papel del profesor como investigador y líder en estos procesos.

Como lo expresan, Ferraro de Velo y Martínez "el avance en investigación educativa universitaria requiere de equipos de investigación multidisciplinares que apliquen procesos de búsqueda de conocimientos, caracterizados por la creatividad e innovación, por los métodos rigurosos utilizados, por el juicio crítico de pares y para mejorar la calidad de la vida en el aula y en la institución" (Ferraro de Velo y Martínez, 2011: 1099).

En Bogotá son tres las universidades que cuentan con programas para licenciados en ciencias naturales (biología, física o química), dos de ellas públicas y una privada, con una duración entre cuatro y cinco años, divididos en 8 o 10 semestres. En ellas proponen, como perfil de sus estudiantes en términos generales, profesionales de la educación con formación investigativa, seres íntegros con valores, conscientes de la importancia social de las ciencias, que piensen y transformen la realidad educativa de sus contextos.

En sus planes de estudio presentan dos ciclos: uno de fundamentación y otro de profundización. En el de fundamentación se propicia la comprensión de los principios básicos de la profesión docente centrada en una disciplina de las ciencias naturales, mientras que el ciclo de profundización está dirigido a espacios académicos referente a las líneas de investigación de los proyectos curriculares específicos. Cabe resaltar que las asignaturas específicas en investigación están en su mayoría en el ciclo de profundización con miras a la inclusión de los estudiantes en investigaciones para el desarrollo de sus trabajos de grado.

De lo anterior surgen los siguientes cuestionamientos: si el propósito de las universidades es una formación de docentes investigadores, ¿por qué en el ciclo de fundamentación no existen espacios académicos para la formación en investigación educativa? ¿por qué no es evidente en las instituciones educativas ese perfil de docente investigador? ¿qué impide que las investigaciones impacten los contextos escolares?

Colombia no es ajena a las limitaciones en la investigación educativa que se plantean en estudios realizados en otros países (como los de Kennedy, 1997; Lüdke, 2005; Miretzky, 2007; Schoonmaker, 2007; Vanderlinde \& Braak, 2010), lo que se requiere en nuestros centros formativos en primera instancia, es el conocimiento de dichas problemáticas para redireccionar los enfoques que se están dando a las investigaciones, de tal forma que trasciendan en los futuros profesores y este proceso se convierta en algo permanente del quehacer profesional en las aulas de clase.

$\mathrm{Al}$ igual, es importante informarse sobre estrategias exitosas realizadas en otros países que sirvan como referentes para proponer soluciones a las limitantes enfrentando de forma más asertiva los problemas de la enseñanza y aprendizaje o como punto de referencia para proponer estrategias innovadoras aplicables a nuestro contexto.

También, es necesario que tanto los profesores de las universidades y los estudiantes generen una mayor cantidad de vínculos profesionales con otras instituciones educativas de diversos niveles y con otros profesionales para conformar comunidades de aprendizaje convirtiéndolas en espacios formativos para la generación de conocimiento y como espacios de discusión y reflexión sobre cuestiones educativas. 


\section{INVESTIGACIONES EDUCATIVAS Y PRÁCTICAS PROFESIONALES EXITOSAS: UNA MIRADA A OTROS CONTEXTOS}

Son múltiples las dificultades que afrontan las instituciones educativas tanto en el proceso de enseñanza como en el de aprendizaje, ejemplo de ello es la falta de interés de los alumnos por aprender, desinterés de los educadores por su formación, pérdida de la calidad en las relaciones generadas dentro de las comunidades educativas, renuencia por aprender ciertas áreas del conocimiento como las ciencias, limitaciones en generar procesos investigativos, dificultades en la comprensión de diferentes saberes, que sin duda, restan valor y desarticulan el proceso formativo que desean ofrecer los centros educativos.

A raíz de estos, se han propuesto alternativas como la formación profesional continua y las comunidades de aprendizaje, en las que los profesores juegan un papel relevante en la transformación de la escuela mediante la inclusión de la investigación como proceso usual en las labores profesionales educativas, "una de las ventajas señaladas por los investigadores que involucran a los profesores en la investigación es la conexión directa que se construye entre la investigación y la práctica" (Schoonmaker, 2007: 268).

Como ejemplo de investigaciones exitosas que dan respuesta a problemáticas educativas se citan las siguientes:

- En Holanda, la colaboración en equipos innovadores (Meirink et al., 2010) permitió el aprendizaje de los profesores cuando trabajan en equipo buscando metas en común, permitiendo la reflexión y transformación de las prácticas para que los estudiantes aprendan de forma activa, regulada y autónoma. En esta estrategia, un ambiente de aprendizaje profesional docente fértil es aquel donde los equipos con un alto nivel de interdependencia y cohesión lograron alinear sus metas para la colaboración, y una proporción relativamente alta de profesores cambiaron sus creencias sobre la enseñanza y el aprendizaje de una manera congruentes con los objetivos propuestos.

- Trent (2010) utilizó un enfoque cualitativo para obtener una comprensión en profundidad del papel que juega la investigación-acción en un grupo de profesores en formación discursiva de inglés en Hong Kong, examinando la forma en que construyen y reconstruyen sus entendimientos y creencias sobre la enseñanza y el aprendizaje. De este trabajo se deduce que se requiere profesores en formación que interpreten y teoricen el significado de la enseñanza y el aprendizaje en términos de diferentes niveles de análisis, incluida la persona que aprende, el aula y la escuela.

- Phelps \& Graham (2010) en Australia desarrollaron una propuesta investigativa a la que denominaron Technology Together, en la cual mediante la investigación acción colaborativa estudiaron la influencia metacognitiva sobre el uso de las TIC por parte de los profesores y las implicaciones de estas para el desarrollo profesional dentro del contexto escolar. El proyecto logró el aumento de la confianza de los profesores en el uso de los computadores; incrementó la integración de las TIC a la enseñanza; apoyó la mejora del currículo e incrementó el diálogo entre profesores dentro de un contexto de la escuela en relación con las TIC.

- Chi (2010), a través de su estudio informa sobre el contenido y el nivel de las reflexiones (descriptivo, interpretativo y crítico) utilizados por doce profesores de inglés en Taiwán, y explora cómo perciben la reflexión en la enseñanza. Los resultados indican que la reflexión puede ayudar a los profesores frente a situaciones 
de incertidumbre, inestabilidad y conflictos en múltiples contextos. Con la ayuda de prácticas reflexivas, algunos maestros cambiaron sus métodos de enseñanza y sus creencias de la enseñanza, ya que lograron comprender mejor las situaciones relacionadas con la enseñanza mediante las reflexiones interpretativas y críticas.

- Tee Ng \& Tan (2009) proponen el empleo de comunidades de práctica para profesores. En las comunidades de práctica se comparten objetivos con el fin de resolver problemas comunes apoyándose en el conocimiento de los demás, mediante el aprendizaje crítico reflexivo.

- Ileana Greca, de la Universidad Luterana en Brasil, y Eduardo González, de la Universidad Nacional de Córdoba en Argentina (2002), formularon la conformación de comunidades de aprendizaje aprovechando el uso de la Internet y las nuevas tecnologías como herramientas útiles para la interacción de redes colaborativas de profesores investigadores, estudiantes y especialistas. Este tipo de trabajo colaborativo implica tanto la construcción de significados por medio de la interacción con otros como el esfuerzo por alcanzar un mismo objetivo, y en el que todos los actores cumplen al mismo tiempo un papel activo y con responsabilidades diversas.

- García, Greca y Meneses (2008) expresan la importancia de comunidades virtuales de práctica para el desarrollo profesional docente en la enseñanza de las ciencias. El potencial que ofrecen las comunidades virtuales puede ser usado en el ámbito educativo, constituyéndolas con el objetivo de mejorar la práctica educativa, creando así un espacio donde las cuestiones relacionadas con el quehacer diario del profesor tengan eco y sean discutidas y compartidas por otros actores implicados en la educación como especialistas en ciencias y didactas de las ciencias.

En las anteriores investigaciones, el educador es eje fundamental en el desarrollo de las mismas, no es solo ejecutor, sino partícipe activo en el proceso para generar conocimientos y cambios en las prácticas educativas. Mediante las prácticas reflexivas los profesores pueden detectar sus fortalezas y debilidades aplicando estrategias que les permitan mejorar las últimas. Pensar, analizar y proponer sobre el ejercicio profesional facilita la discusión entre pares para llegar a soluciones donde el trabajo en equipo de forma colaborativa llega a convertirse en un factor decisivo en el éxito o fracaso de las estrategias seguidas por los equipos.

De tal forma, el desarrollo de propuestas innovadoras en educación y su efectiva transferencia al aula puede ser más eficaz como resultado del trabajo de una comunidad en la que participan personas con funciones diversas, pero todas imprescindibles e interrelacionadas, que comparten objetivos y planteamientos conceptuales. Estas comunidades posibilitan a los participantes acercarse y construir conjuntamente conocimiento.

También, el uso de las TIC son una herramienta que facilita la comunicación entre profesores, alumnos o especialistas para establecer comunidades de aprendizaje, comunidades de práctica y redes colaborativas en las que la discusión y reflexión sobre cuestiones educativas alcanza niveles en los que las transformaciones sobre el propio aprendizaje y la enseñanza trascienden a las aulas de clase de forma positiva.

Son muchos los ejemplos y propuestas que sirven como referentes en los que la investigación presenta resultados propicios para responder a las necesidades de la educación como sistema que logre la formación de personas capaces de generar conocimiento para 
transformar sus entornos, demostrando valores en una sociedad cambiante y que requiere de todos la adaptación a las exigencias diarias.

\section{DISCUSIONES FINALES}

La investigación educativa es un proceso de formación continua, del que el profesor enriquece sus conocimientos teóricos y los aplica en las prácticas. La sociedad que está en constante cambio requiere profesionales en educación que transformen sus prácticas para lograr la formación de sus educandos acorde a las necesidades del contexto. En este sentido, "el ejercicio profesional docente, asumido como un ejercicio de profesionalización, pasaría de la expresión de acciones y rutinas, a convertirse en una praxis, intencionada, generadora de sentidos y de saberes docentes" Núñez, Arévalo y Ávalos (2012: 13), es decir, las prácticas también han de ser espacios académicos que permitan al profesor reflexionar y aprender.

Es así, que gracias a los procesos de investigación educativa, el docente adquiere destrezas y habilidades que le permiten construir conocimiento, cambiar sus discursos, metodología y métodos usados en las intervenciones directas e indirectas en las clases, transformando no solo sus conocimientos (profesional, base y práctico), sino también sus procesos de enseñanza, fomentando en los educandos a su vez conocimientos que puedan ser usados en los contextos particulares para los que se están formando.

La primera barrera a vencer, como agentes activos de la educación, es una mayor participación en lo referente a la mejora no solo de conocimientos mediante una formación continua, también es importante un mayor esfuerzo y dedicación en cuanto a la búsqueda de estrategias que permitan solucionar las dificultades que afectan el aprendizaje de los educandos.

Las instituciones de todos los niveles de educación tendrán que buscar mayores vínculos entre ellas para fomentar la enseñanza y aprendizaje tanto de profesores como de alumnos, donde la colaboración sea un recurso que posibilite el logro de metas comunes para proponer soluciones a las problemáticas de la educación mediante el análisis, la discusión fundamentada, la creatividad y la innovación.

El profesor es, por tanto, el articulador entre la teoría y la práctica educativa desempeñando el papel protagónico en la mejora de la profesionalidad docente $y$, por tanto, de la educación, siendo el intermediario entre la investigación y la práctica, empoderándose de todo lo que conlleva el ejercicio de la enseñanza, donde involucre su conocimiento base, profesional y práctico, y al igual que le permita emanciparse de sus viejos hábitos donde se limita a dar a conocer sus saberes sin que ellos trasciendan en sus estudiantes.

\section{REFERENCIAS BIBLIOGRÁFICAS}

Black, P. \& Plowright, D. (2010). A multi-dimensional model of reflective learning for professional development. Reflective Practice, vol. 11 (2), 245-258.

Cochran-Smith, M. \& Lytle, S. (2009). The teacher research movement: A decade later. Educational Researcher, vol. 28 (7), 15-25.

Chi, F. (2010). Reflection as teaching inquiry: Examples from Taiwanese in-service teachers. Reflective Practice, vol. 11 (2), 171-183.

Delorenzi, O., Pacheco, L., Seoane, V., Otonello, L. y Scempio, V. (2004). La investigación acción emancipatoria en la práctica docente: Resultados, problemas y reflexiones. I Congreso 
Internacional Educación, Lenguaje y Sociedad. Tensiones educativas en América Latina. La Pampa, Argentina.

Ferraro de Velo, A. y Martínez, A. (2011). La formación en investigación en la profesionalización docente. Actas del III Congreso Internacional de Nuevas Tendencias en la Formación Permanente del Profesorado (pp. 1095-1100). Barcelona: Universidad de Barcelona.

Garay, F., (2013) La naturaleza del conocimiento químico en la educación en química. Revista Chilena de Educación Científica, vol. 12 (1), 17-21.

García, J., Greca, I. y Meneses, J. (2008). Comunidades virtuales de práctica para el desarrollo profesional docente en enseñanza de las ciencias. Revista Electrónica de Enseñanza de las Ciencias, vol. 7 (2), 439-462.

Greca, I. y González, E. (2002). Comunidades de aprendizaje en desarrollo sustentable. Actas de XX Encuentros de Didáctica de las Ciencias Experimentales (pp. 231-238). La Laguna: Universidad de La Laguna.

Hiebert, J., Gallimore, R. \& Stigler, J. (2002). A knowledge base for the teaching profession: What would it look like and how can we get one? Educational Researcher, vol. 31 (5), 3-15.

Imbernón, F., Alonso, M., Arandia, M., Cases, I., Cordero, G., Fernández, I., Revenga, A. y Ruíz, P. (2007). La investigación educativa como herramienta de formación del profesorado. Reflexión y experiencias de investigación educativa. Barcelona: Editorial Graò.

Kennedy, M. (2002). Knowledge and teaching. Teachers and Teaching: Theory and Practice, vol. 8 (3), 355-370.

(7), 4-12. (1997). The connection between research and practice. Educational Researcher, vol. 26

Lüdke, M. (2005). O profesor e sua formaçáo para a pesquisa. Educação \& Sociedade, vol. XXII (74), 333-349.

Maciel de Oliveira, C. (2003). Investigar, reflexionar y actuar en la práctica docente. Revista Iberoamericana de Educación, (33). Recuperado el 1 de octubre de 2013 desde http://www.rieoei. org/rie_contenedor.php?numero=inv_edu2\&titulo=Investigar,\%20reflexionar\%20y\%20actuar\%20 en\%20la\%20pr\%E1ctica\%20docente

Martínez González, R. A. (2007). La investigación en la práctica educativa: Guía metodológica de investigación para el diagnóstico y evaluación en los centros docentes. Madrid: Centro de Investigación y Documentación Educativa, Ministerio de Educación y Ciencia.

Meirink, J., Imants, J., Meijer, P. \& Verloop, N. (2010). Teacher learning and collaboration in innovative teams. Cambridge Journal of Education, vol. 40 (2), 161-181.

Miretzky, D. (2007). A view of research from practice: Voices of teachers. Theory into Practice, vol. 46 (4), 272-280.

Núñez, M., Arévalo, A. y Ávalos, B. (2012). Profesionalización docente: ¿Es posible un camino de convergencia para expertos y novatos? Revista Electrónica de Investigación Educativa, vol. 14 (2), 10-24.

Phelps, R. \& Graham, A. (2010). Exploring the complementarities between complexity and action research: The story of technology together. Cambridge Journal of Education, vol. 40 (2), 183-197.

Vanderlinde, R. \& Braak, J. (2010). The gap between educational research and practice: Views of teachers, school leaders, intermediaries and researchers. British Educational Research Journal, vol. 36 (2), 299-316.

Schoonmaker, F. (2007). One size doesn't fit all: Reopening discussion of the research-practice connection. Theory into Practice, vol. 46 (4), 264-271.

Tee Ng, P. \& Tan, C. (2009). Community of practice for teachers: Sensemaking or critical reflective learning? Reflective Practice, vol. 10 (1), 37-44.

Trent, J. (2010). Teacher education as identity construction: Insights from action research. Journal of Education for Teaching, vol. 36 (2), 153-168. 
